\title{
The Seebeck Effect in Double Tunnel Junctions with Ferromagnetic Electrodes and Central Layer Separated by Nonmagnetic Barriers
}

\author{
M. WILCZYŃSKI* \\ Faculty of Physics, Warsaw University of Technology, Koszykowa 75, 00-662 Warsaw, Poland
}

\begin{abstract}
The Seebeck effect is analysed in the double planar tunnel junctions consisting of ferromagnetic electrodes and the central layer separated by nonmagnetic barriers with the arbitrary angle between magnetic moments in neighbouring ferromagnetic layers. The Seebeck coefficient is calculated as a function of the thickness of the central layer. The influence of temperature of the junction and the relative orientation of magnetic moments in ferromagnetic layers on this coefficient is also analysed. Calculations are performed in the linear response theory using the free-electron model. It has been found that the Seebeck coefficient oscillates with the thickness of the central layer and can be strongly enhanced in the junction with special central layer thickness due to electron tunnelling by resonant states. The form of the observed oscillations depends on the temperature of the junction. The magnitude of the Seebeck coefficient usually increases with the increase of the angle between magnetic moments in the neighbouring ferromagnetic layers as in the case of single junctions. However, in the junctions with the specially designated central layer the decrease of the magnitude of the Seebeck coefficient with the increase of this angle can be observed.
\end{abstract}

DOI: $10.12693 /$ APhysPolA.133.544

PACS/topics: 72.20.Pa, 73.40.Gk,73.40.Ty, 73.50.Lw

\section{Introduction}

The investigations concerning the different thermoelectric effects in low-dimensional systems have recently attracted much attention. Among them there is the Seebeck effect (thermopower), which is related to the appearance of the bias voltage across the system when the thermal gradient is applied to it. This effect has been experimentally detected e.g. in the tunnel planar junctions consisting of CoFe electrodes and $\mathrm{MgO}$ barrier (e.g. [1]), showing spin-polarized electron transport. The experimental determination of the Seebeck effect in such systems encourages theoretical investigations concerning the similar systems. The appropriate calculations are performed using the simple free-electron models (e.g. [2-4]) as well as ab-initio formalism (e.g. [5, 6]). The Seebeck effect in such systems depends significantly on the magnetic configuration of the junctions [1-6], so potentially it can be used in constructing various spintronic devices. It is known that many effects connected with electron transport in single tunnel junctions can be slightly enhanced in double tunnel junctions due to the resonant tunnelling through such systems by resonant states. This enhancement is especially significant in the junctions in which individual resonant states can be active in tunnelling. Such situation appears in double tunnel junctions with the electrodes and the narrow central layer made of semiconducting materials. Such junctions can be epitaxially grown with abrupt interface and generally

*corresponding author; e-mail: wilczyns@if.pw.edu.pl can be more easily integrated with other semiconductor devices. When the central layer has ferromagnetic properties one can change the position of resonant states using the external magnetic field, which enables the use of such field to modify the electric and thermoelectric properties of the junction.

In the present paper the thermoelectric (Seebeck) effect in double planar tunnel junctions consisting of external electrodes and the central layer made of semiconducting ferromagnets separated by two nonmagnetic barriers is analysed. These barriers can be made from such materials as $\mathrm{AlO}_{x}[7], \mathrm{AlAs}$ [8] or GaAs [9] whereas the electrodes can be made from such materials as GaMnAs [8,9] or $\mathrm{TiCoO}[7]$. The Curie temperature of GaMnAs is usually relatively small $\left(T_{\mathrm{C}} \approx 100 \mathrm{~K}\right)$, however recently $T_{\mathrm{C}}=185 \mathrm{~K}$ is detected in such films after the annealing [10]. In $\mathrm{TiCoO}$ films the Curie temperature can reach even $T_{\mathrm{C}}=400 \mathrm{~K}$. In the single junction of $\mathrm{FeCo} / \mathrm{Al}_{2} \mathrm{O}_{3} / \mathrm{TiCoO}$ type the tunnel magnetoresistance effect has been observed in the temperatures reaching $T_{\mathrm{C}}=180 \mathrm{~K}$ [7]. Taking this into account one can expect that the Seebeck effect in such systems can also depend on the magnetic configuration of the junction.

\section{Model}

The double tunnel junction consisting of external ferromagnetic electrodes and the central ferromagnetic layer separated by two nonmagnetic barriers of equal thickness is considered. It is assumed that magnetic moments in the external electrodes are aligned in the parallel way, whereas the magnetic moment of the central layer can form the arbitrary angle with the moments of the external electrodes. The calculations of the Seebeck coefficient 
$S$ are performed in the linear response theory assuming the free-electron-like spin-polarized one-band model to describe electron structure in the conducting layers. The Seebeck coefficient is generally related to the voltage drop $\Delta V$ across the junction induced by the temperature difference $\Delta T$ between the electrodes on condition that the charge current vanishes. This leads to the well-known formula for $S$

$$
S=\lim _{\Delta T \rightarrow 0} \frac{\Delta V}{\Delta T}=-\frac{1}{e T} \frac{L_{1}}{L_{0}} .
$$

In the equation (1) the quantity $L_{n}(n=0,1)$ can be calculated from the formula [2]:

$$
\begin{aligned}
L_{n} & =-\frac{2 \pi m}{h^{3}} \sum_{\sigma} \int \mathrm{d} \varepsilon_{\|} \int \mathrm{d} \varepsilon_{\perp}\left(E-E_{\mathrm{F}}\right)^{n} W_{\sigma}\left(\varepsilon_{\perp}\right) \\
& \times \frac{\mathrm{d} f(E, T)}{\mathrm{d} E} .
\end{aligned}
$$

The Fermi-Dirac distribution function $f(E, T)$ depends on the average junction temperature $T$ and the total energy $E$ of the tunnelling electrons whereas transmission coefficient of such electrons $W_{\sigma}\left(\varepsilon_{\perp}\right)$ depends on the energy $\varepsilon_{\perp}$ connected with their movement in the direction perpendicular to the interfaces. Summation in the equation (2) is done over two possible orientations of the electron spin in the external electrodes, whereas integration is performed over energies $\varepsilon_{\perp}$ and $\varepsilon_{\|}=E-\varepsilon_{\perp}$. The coefficient $W_{\sigma}\left(\varepsilon_{\perp}\right)$ is calculated assuming coherent regime of electron transport through the whole system. Due to the noncollinear arrangement of magnetic moments in the external electrodes and the central layer the appropriate spinor transformations are applied in formulating the matching conditions of the electron wave functions at the interfaces.

\section{Results and conclusions}

The schematic potential profile for electrons of both direction of spin in the double tunnel junction with the parallel configuration of magnetic moments in the electrodes and the central layer is presented in Fig. 1. The numerical calculations of the Seebeck coefficient are performed for the following parameters: the Fermi energy $E_{\mathrm{F}}=0.16 \mathrm{eV}$, spin-splitting of the electron bands in the ferromagnetic electrodes and the central layer $2 \Delta=$ $0.08 \mathrm{eV}$, the height of the barriers $U=0.47 \mathrm{eV}$ and their thickness $d_{b}=0.6 \mathrm{~nm}$. Such parameters can correspond roughly to the GaMnAs/AlAs systems [8].

In Fig. 2 the Seebeck coefficient is presented for three different temperatures as a function of the central layer thickness. The results of the calculations performed for the parallel and the antiparallel configuration of magnetic moments in the external electrodes and the central layer are presented in Figs 2a-b and 2c-d respectively. The horizontal lines in these figures correspond to values of the Seebeck coefficient obtained in the single planar junction with the parallel (Fig. 2a,b) and antiparallel (Fig. 2c,d) configuration of magnetic moments in the electrodes.

As one can see the Seebeck coefficient in double junctions depends strongly on the central layer thickness $d_{c}$.

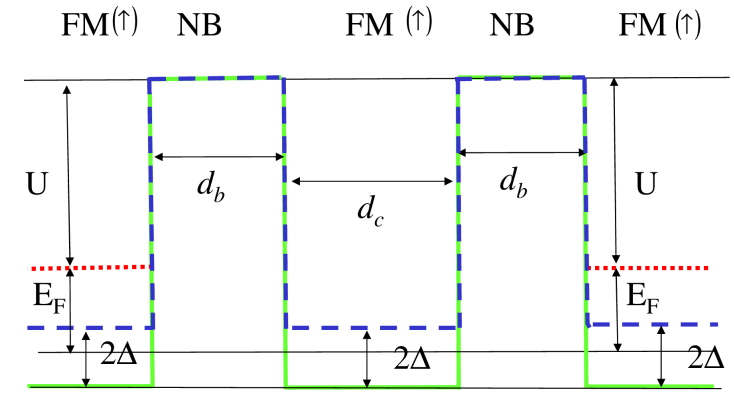

Fig. 1. The schematic potential profile for spin-up (solid line) and spin-down (dashed line) electrons for the parallel configuration of magnetic moments in the external electrodes and the central layer. The dotted line denotes the position of the Fermi level in the electrodes in the limit of vanishing voltage drop across the junction.

The magnitude of the Seebeck coefficient obtained in the double junction averaged over the thickness of the central layer is similar to the magnitude of this coefficient obtained in the single junction. However, it can be significantly enhanced in the junctions of the special $d_{c}$. This enhancement can be related to electron tunnelling by resonant quantum-well states in the central layer. The observed peaks are especially high in the junctions with the narrow central layer in which individual resonant states are active in tunnelling. The height and width of the discussed peaks increase with the increase of the temperature of the junction. This is connected with the larger smoothing of the Fermi-Dirac distribution function in higher temperatures. Additionally the peaks in Fig. 2 slightly move towards a smaller central layer thickness $d_{c}$ with the increase of the junction temperature $T$. However the shift of the position of the peaks with the increase of $T$ is small and can be observed only in Fig. 2a and Fig. 2c where the axis scale is taken in such a way that only one peak can be observed. The shift of the position of the similar peak towards a narrower central layer thickness has been also detected in double junctions with nonmagnetic electrodes and ferromagnetic barriers [4].

The thickness of the central layer for which the enhancement of the Seebeck coefficient is observed does not depend significantly on the orientation of magnetic moments in the central layer. This can be connected with the fact that the discussed enhancement is always obtained when the resonant levels for spin-up or spin-down electrons are located slightly above the Fermi level in the external electrodes. Usually such levels in the systems specified by the parameters taken in the calculations are active in electron transport despite the actual angle between magnetic moments in external electrodes and the central layer, which leads to the simultaneous increase of the Seebeck coefficient for all magnetic configurations of the junction. However, more detailed analysis of the Seebeck coefficient versus the angle $\theta$ between magnetic moments in external electrodes and the central layer in- 

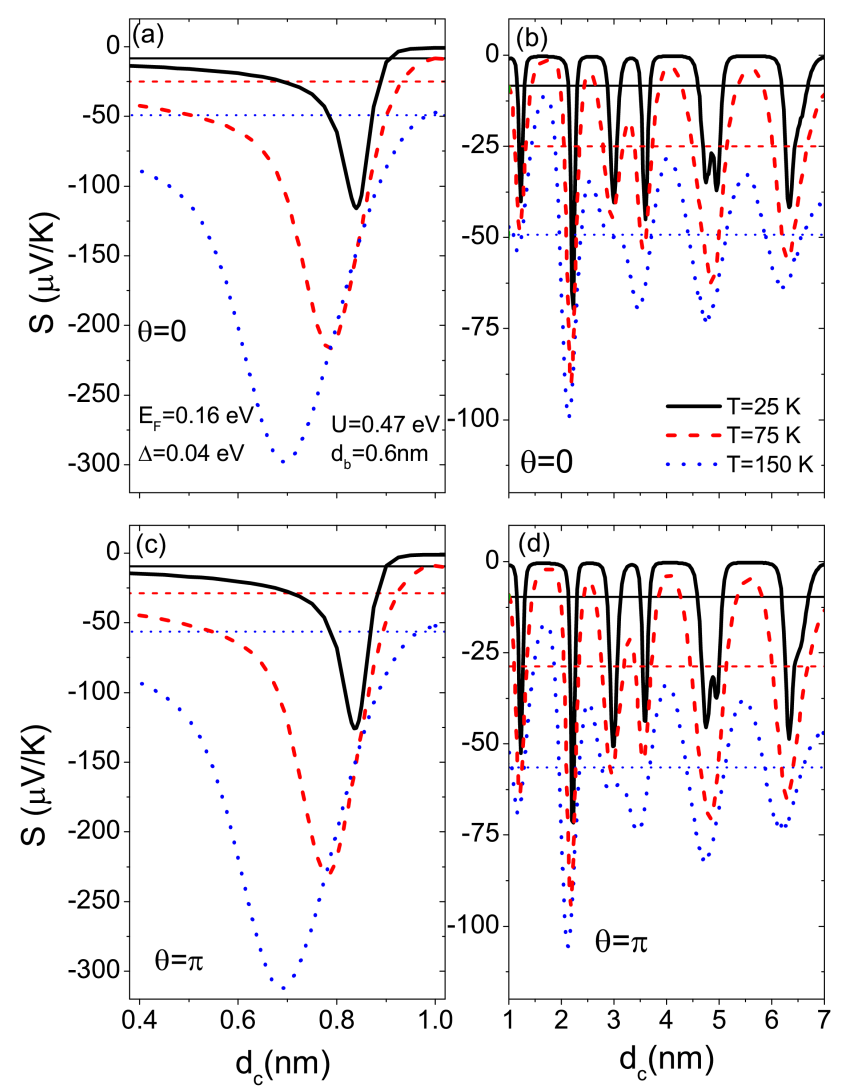

Fig. 2. The Seebeck coefficient in the double tunnel junction as a function of the thickness of the central layer $d_{c}$ obtained for the indicated values of the angle between magnetic moment in the central and external ferromagnetic layers $\theta=0(\mathrm{a}, \mathrm{b})$ and $\theta=\pi(\mathrm{c}, \mathrm{d})$ and for three indicated temperatures $T$ of the junction. The parameters of the junction are: the Fermi energy $E_{\mathrm{F}}=0.16 \mathrm{eV}$, the spin-splitting of the electron bands in the central layer and the external electrodes $2 \Delta=0.08 \mathrm{eV}$, the height and the width of both barriers $U=0.47 \mathrm{eV}$ and $d_{b}=0.6 \mathrm{~nm}$ respectively. The horizontal lines correspond to the results obtained for the single junction with the same ferromagnetic electrodes and the nonmagnetic barrier as in the double junction for the specified angles between magnetic moments in the electrodes. Parts $2 \mathrm{a}-\mathrm{c}$ and $2 \mathrm{~b}-\mathrm{d}$ differ in the range of $d_{c}$ changes.

dicates the significant influence of the central layer thickness on the character of the angular dependence of Seebeck coefficient.

The discussed dependence can be analysed in Fig. 3ac where the ratio of the Seebeck coefficient obtained for the specified magnetic configuration specified by the angle $\theta$ to this coefficient obtained in the parallel configuration is presented as a function of the angle $\theta$ between magnetic moments. Results presented in Fig. $3 \mathrm{~b}$ in which the decrease of the magnitude of this coefficient with the increase of the angle $\theta$ is observed are obtained for $d_{c}=0.9 \mathrm{~nm}$, which is larger than the central layer thickness for which the resonant enhancement

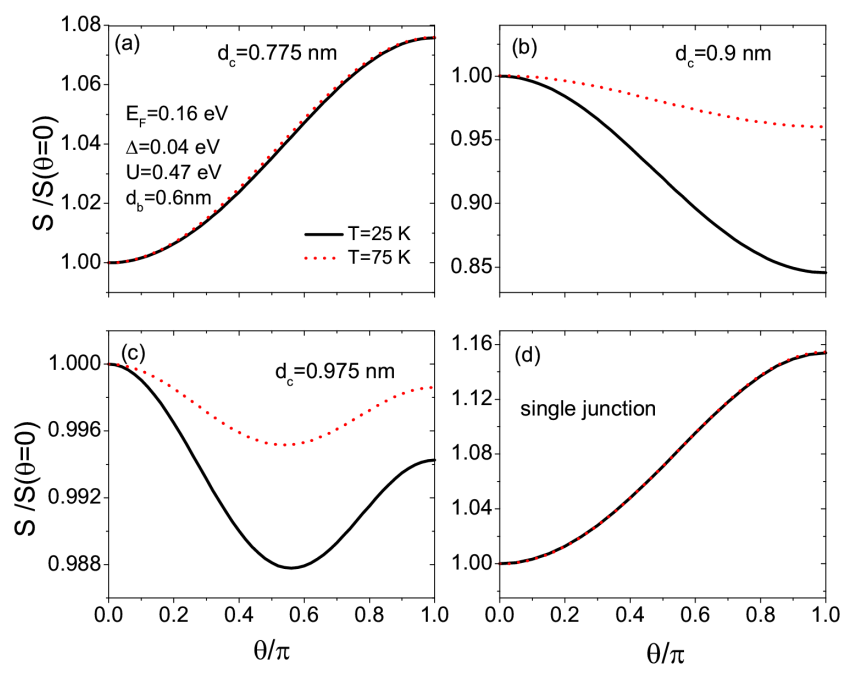

Fig. 3. The Seebeck coefficient as a function of angle between magnetic moments in the neighbouring ferromagnetic layers in double (a-c) and single (d) tunnel junctions obtained for two different indicated values of temperature of the junction $T$. The $d_{c}$ in parts a-c denotes the thickness of the central layer. The other parameters of the junctions are the same as in Fig. 2.

of the Seebeck coefficient is obtained. In the junctions with slightly wider central layer the Seebeck coefficient can vary non-monotonically with the increase of $\theta$ which can be observed in Fig. 3c presenting the results obtained for $d_{c}=0.975 \mathrm{~nm}$.

When $d_{c}$ is assumed in a different way and correspond either to the position of the peaks in Fig. 2a or to the case when $d_{c}$ is smaller than the central layer thickness corresponding to the position of these peaks the monotonous increase of magnitude of the Seebeck coefficient with the increase of the angle $\theta$ is observed, which can be seen in Fig. 3a. Similar types of dependence are obtained in the single junction with the barrier and electrodes made of the same materials as in the case of the double junction, which can be seen in Fig. 3d. Such behaviour is characteristic for the Seebeck coefficient in junctions with barriers much higher than the width of the electron bands in the electrodes [2]. Although the normalized Seebeck coefficient obtained in double junctions can change with the change of $\theta$ slightly weaker than in single junctions, the absolute difference between magnitudes of this coefficient in the parallel and antiparallel configurations is strongly enhanced in the limit of resonant tunnelling in double junctions due to the strong enhancement of Seebeck coefficient.

\section{References}

[1] N. Liebing, S. Serrano-Guisan, K. Rott, G. Reiss, J. Langer, B. Ocker, H.W. Schumacher, J. Appl. Phys. 111, 07C520 (2012).

[2] M. Wilczynski, J. Phys.: Condens. Matter 23, 456001 (2011). 
[3] C. López-Monís, A. Matos-Abiague, J. Fabian, Phys. Rev. B 89, 054419 (2014).

[4] M. Wilczyński, J. Magn. Magn. Mater. 421, 418 (2017).

[5] M. Czerner, M. Bachmann, Ch. Heiliger, Phys. Rev. B 83, 132405 (2011).

[6] S.-Z. Wang, K. Xia, G.E.W. Bauer, Phys. Rev. B 90, 224406 (2014).

[7] H. Toyosaki, T. Fukumura, K. Ueno, M. Nakano, M. Kawasaki, Jpn. J. Appl. Phys. 44, L896 (2005).
[8] M. Tanaka, Y. Higo, Phys Rev. Lett. 87, 026602 (2001).

[9] D. Chiba, F. Matsukura, H. Ohno, Physica E 21, 966 (2004).

[10] V. Novák et al, Phys. Rev. Lett. 101, 077201 (2008). 